\title{
boulons de mesures équipés de jauges extensométriques essais en laboratoire et in situ
}

\author{
par \\ R. Poirot \\ Ingénieur au CERCHAR \\ (Centre d'Etudes et de Recherches des Charbonnages de France)
}

L'étude du comportement d'un massif rocheux renforcé par un boulonnage réparti - à la résine ou au ciment - peut être abordée en suivant les déformations des boulons au moyen de jauges extensométriques.

Cette méthode a déjà été utilisée [1], mais l'expérience a montré que la conception des boulons de mesure mis en œuvre n'était pas satisfaisante : les jauges collées sur la paroi extérieure étaient vulnérables $(50 \%$ des jauges étaient détériorées à la mise en place) et la préparation de leur emplacement impliquait une réduction notable de la section de leur emplacement impliquait une réduction notable de la section de telle sorte que sous traction la zone de mesure entrait en déformation plastique alors que le reste du boulon restait en déformation élastique.

\section{Description du boulon CERCHAR - figure 1}

Pour éviter les inconvénients cités, nous avons collé les jauges dans une cavité de section carrée et coaxiale aux boulons. Les jauges sont naturellement protégées et les fils, raccordés directement à une boîte de jonction placée à l'extrémité du boulon, ne risquent pas d'être détériorés ni
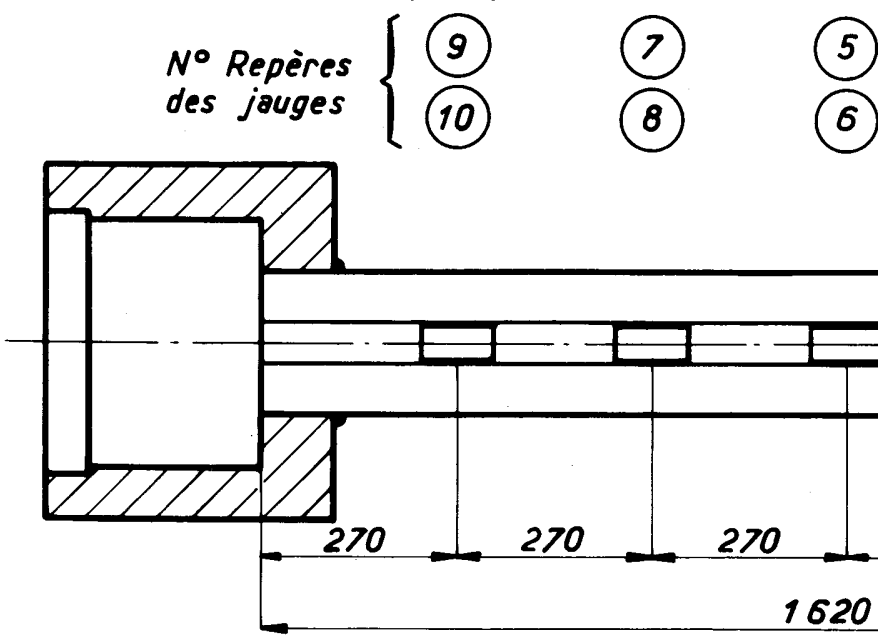

attaqués par les produits de scellement.

La section droite garde une section constante de telle sorte que les jauges rendent bien compte de la déformation d'un point courant du boulon.

Les jauges sont groupées par paires collées face à face. A l'origine nous avons retenu cette disposition pour remédier aux défaillances rencontrées antérieurement, chaque jauge pouvant être interrogée individuellement ou en combinaison avec sa voisine.

Les essais au laboratoire, puis au chantier, ayant donné un fonctionnement correct de toutes les jauges, nous déterminons la traction exercée en faisant la somme des indications et nous obtenons une indication sur la courbure par difference.

\section{Essais au laboratoire}

\subsection{Essai de traction}

Les boulons d'essais ont été montés sur une machine de traction et les déformations suivies simultanément par les jauges et un comparateur classique installé dans la partie médiane sur une base de $350 \mathrm{~mm}$.

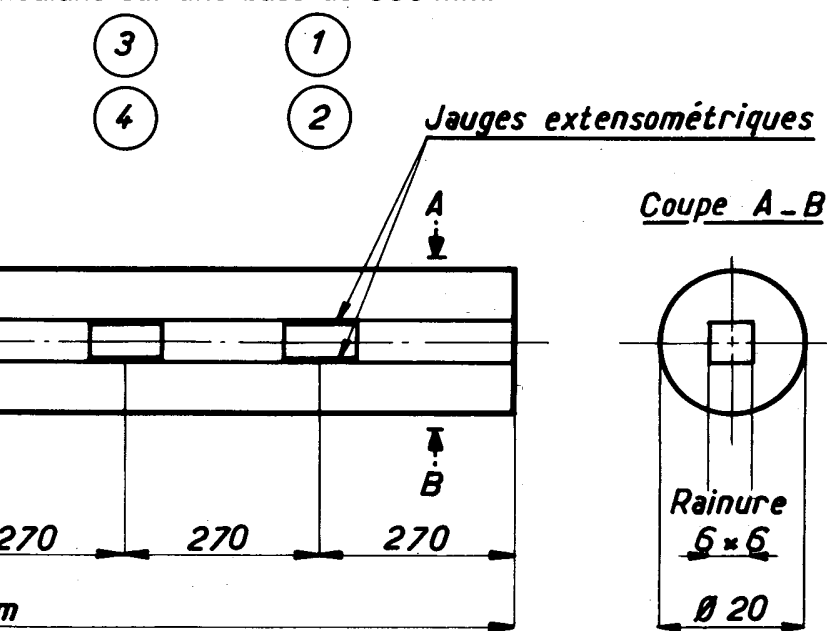

Fig. 1 Boulon de mesure - Coupe longitudinale 


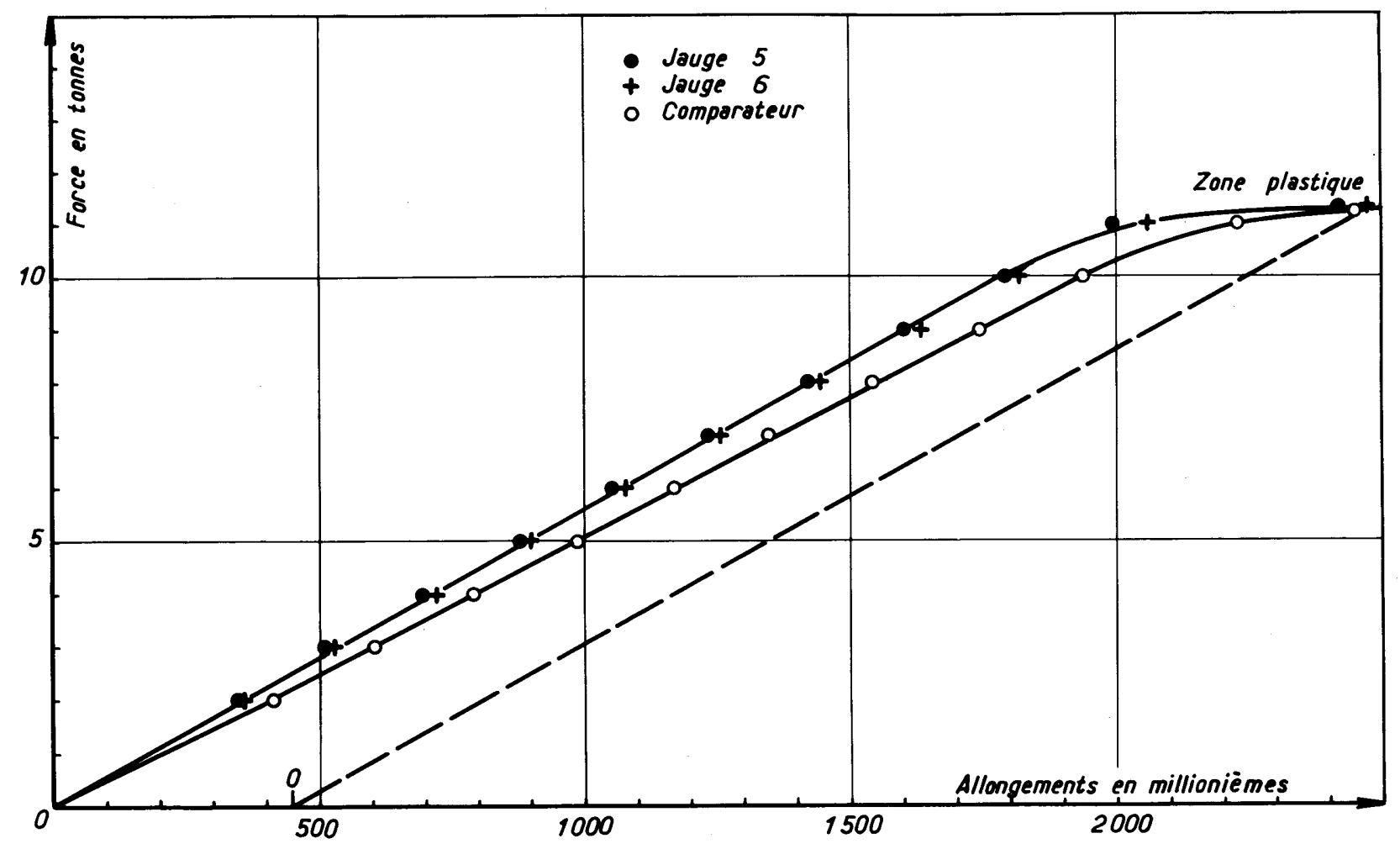

Fig. 2 Etalonnage en traction

Fig. 3 Etalonnage en flexion

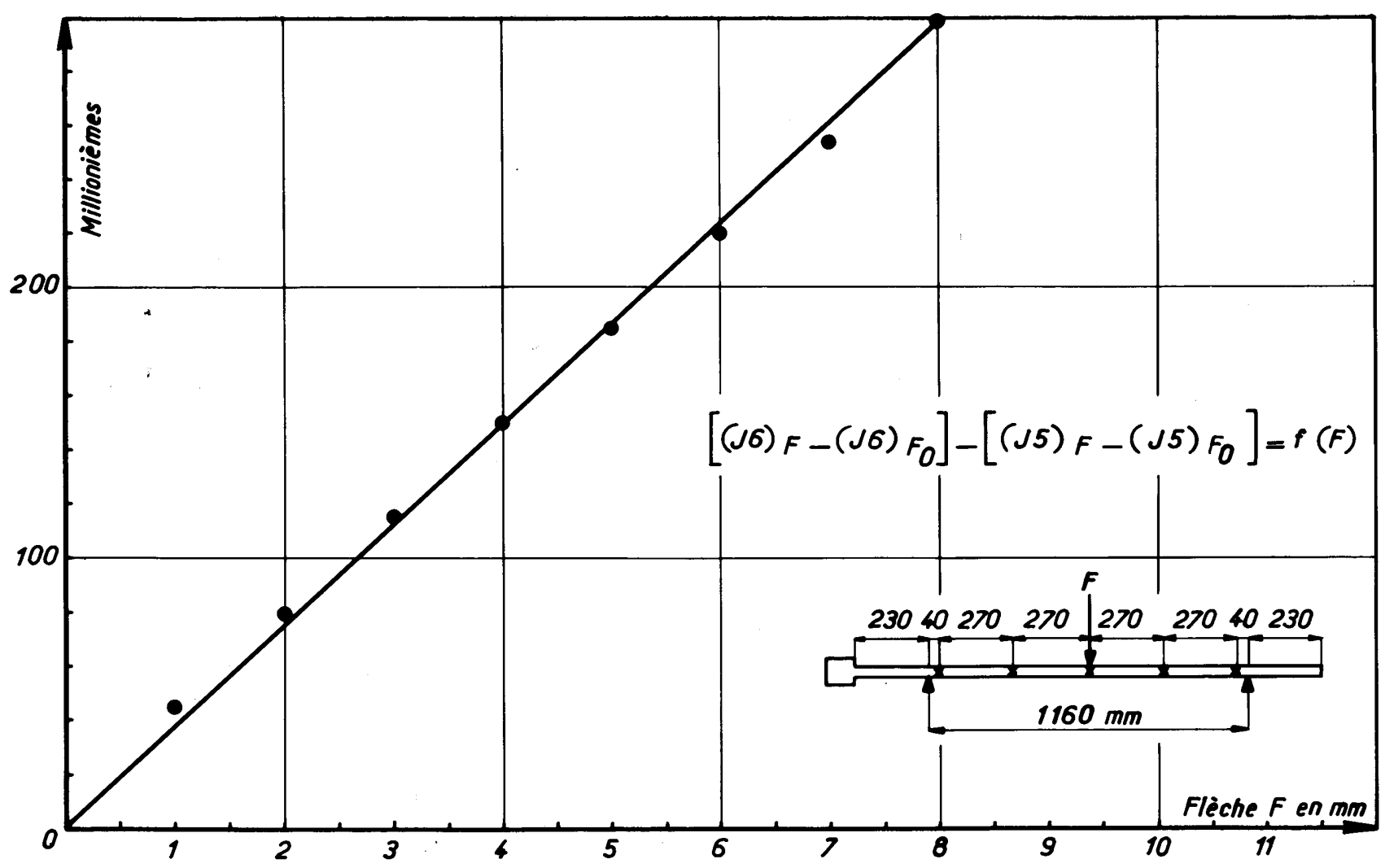




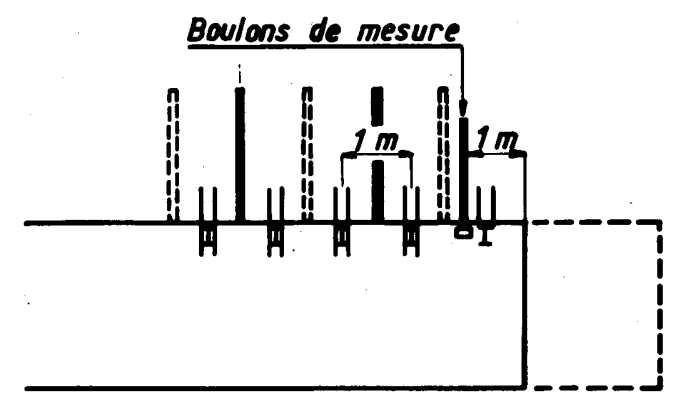

Abottage $2 \mathrm{~m}$ (tir 2,40 m)

Profondeur : $1050 \mathrm{~m}$

Puissance de $/ 0$ veine : $2 \mathrm{~m}$

Voie trapézoîdale : $6 \times 2,50 \mathrm{~m}$

Boulons : $2,20 m-1,3$ boulon $/ m^{2}$

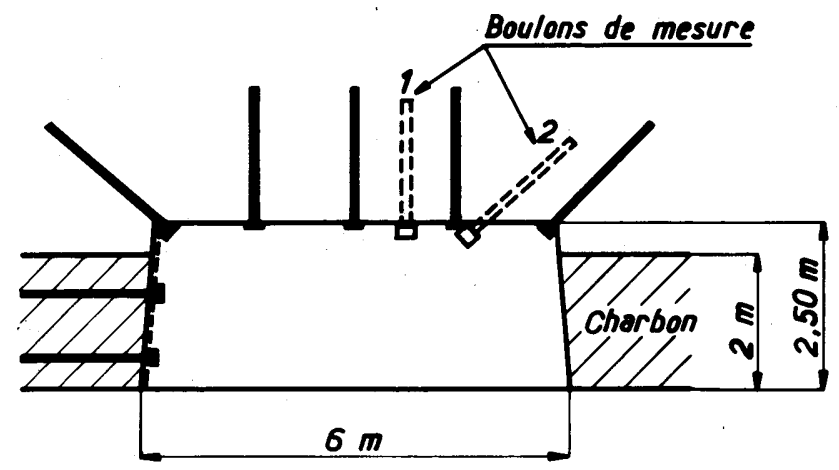

Vue en plon

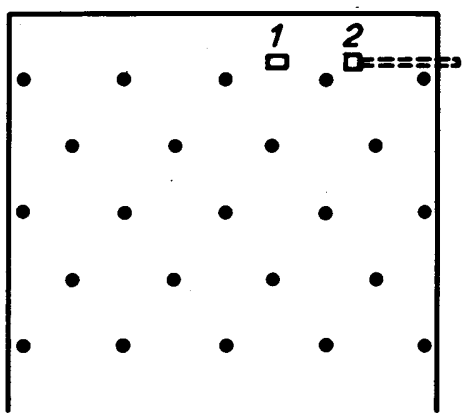

Fig. 4 Wendel - veine 27/28 - Panneau 5

Schéma du boulonnage et implantation des boulons de mesure

La figure 2 donne un résultat partiel des essais jusqu'à un seuil de déformation voisin de $2 / 1000$, les jauges montrent un comportement élastique du boulon. L'écart entre leurs indications et celles du comparateur (d'environ $4 \%$ ) pourrait être corrigé car il vient du fait que nous avons adopté le facteur de jauge indiqué par le constructeur. Pour le moment nous ne jugeons pas nécessaire de déterminer ce facteur pour chaque jauge et l'erreur qui en résulte entre dans la dispersion des résultats. A titre indicatif, l'écart-type des variations de longueur correspondant à des augmentations de traction de 1 tonne (de 0 a 10 tonnes, soit 100 mesures pour un boulon) est de 5 millionièmes* pour une variation moyenne de 182 millionièmes par tonne.

\subsection{Essais de flexion}

Le boulon, placé sur deux appuis simples, a été sollicité par une force variable appliquée en face d'un groupement de jauges - jauges 5 et 6 .

Les essais ont été faits avec et sans effort axial.

La figure 3 donne la correspondance entre la flèche mesurée et la différence entre les variations des indications des deux jauges placées face à face.

L'ensemble des essais a montré une bonne résistance des jauges à diverses sollicitations et la possibilité d'interpréter les valeurs mesurées en vue de décrire des déformations en flexion plane au voisinage des jauges.

\section{Essais au chantier}

Nous avons placé deux boulons de mesure dans une section boulonnée d'une galerie minière. Ils furent scellés à la résine comme les autres boulons. La figure 4 indique leurs positions.

Les terrains dont la coupe est donnée par la figure 5 sont de qualité médiocre et relativement hétérogènes. De plus, ils sont soumis à des contraintes élevées (profondeur $1050 \mathrm{~m})$, de l'ordre de 200 bars. Une observation endoscopique avait montré la présence de trois fissures dont une ouverte à $70 \mathrm{~cm}$ de la paroi de la galerie.

- Un millionième est un allongement relatif de 10-6. Il est souvent appelé micro-déformation $(\mu \mathrm{d})$ par analogie avec le terme anglais microstrain.

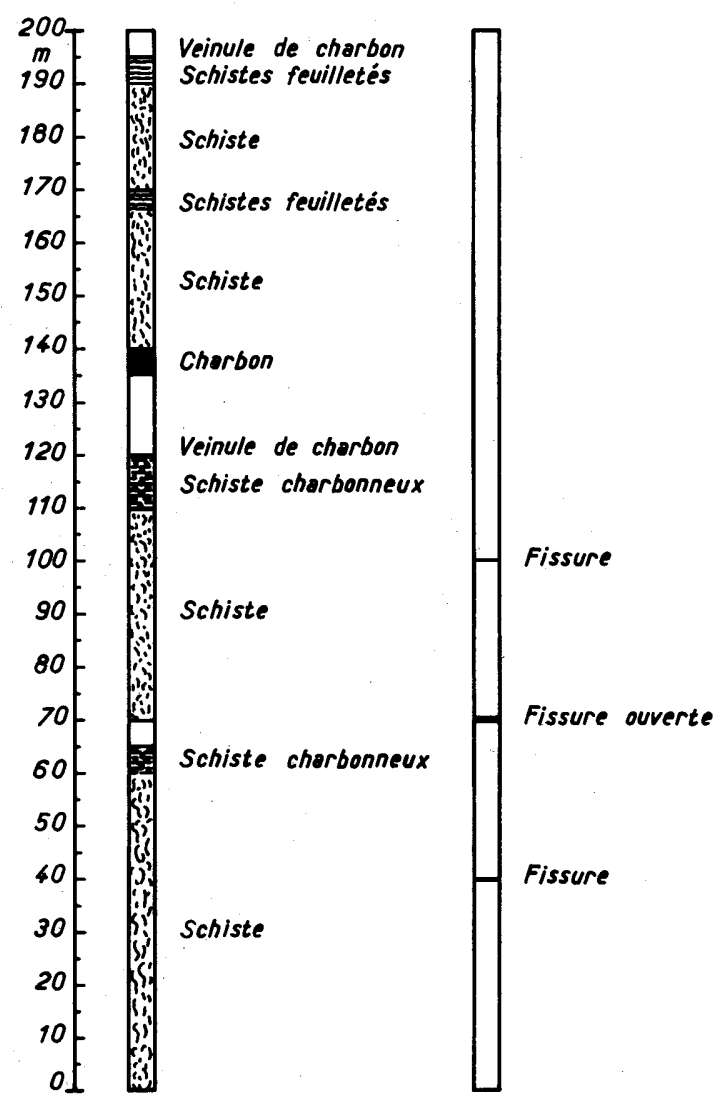

Noture des terrains

Fissuration relevée ì l'endoscope

Fig. 5 Wendel - veine 27/28 - Panneau 5

Descenderie de taille. Nature du toit 


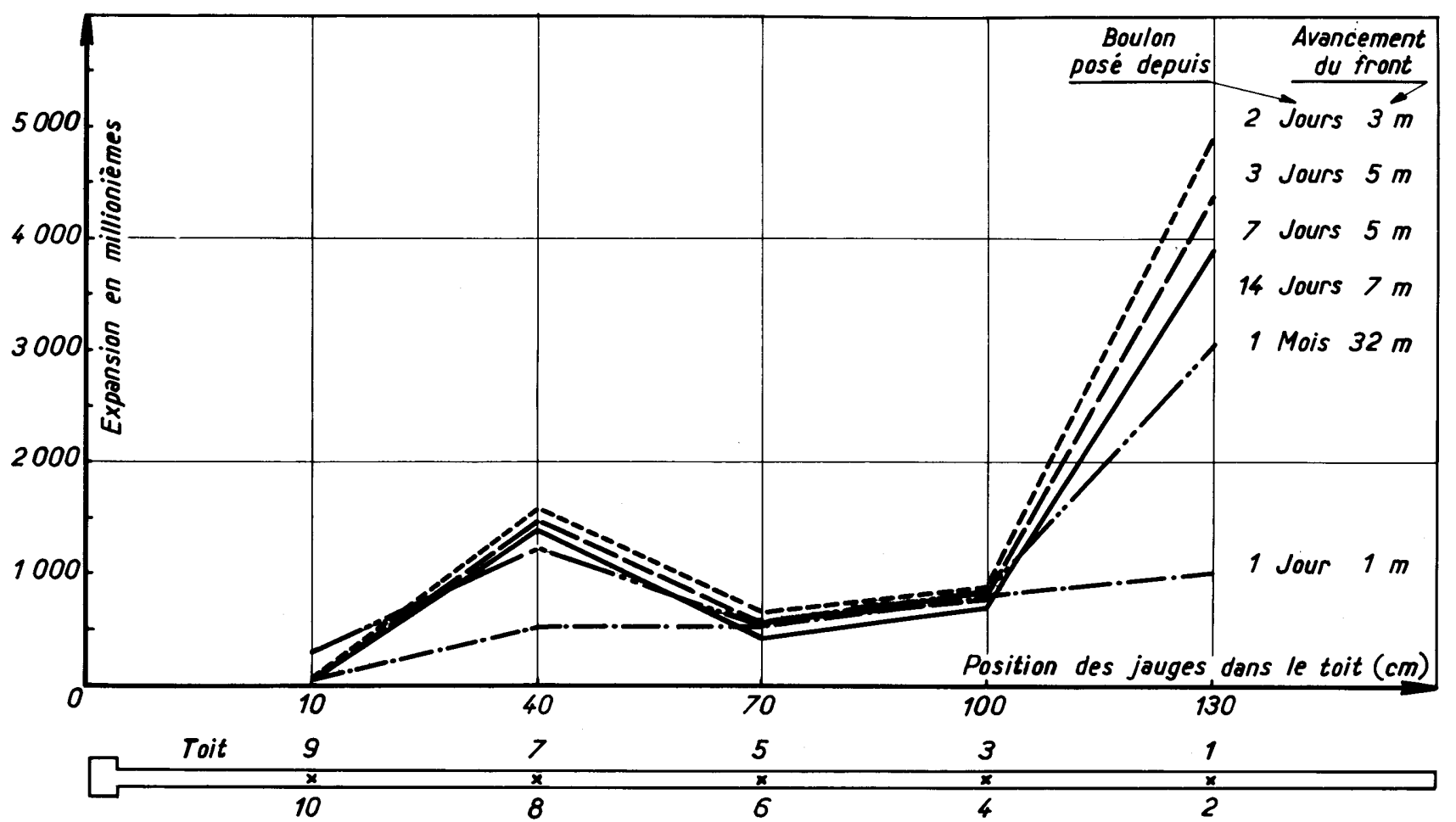

Fig. 6 Wendel - veine 27/28 - Panneau 5

Boulon de mesure $n^{\circ}$ 1. Allongement en fonction du temps et de l'avancement du front

La figure 6 donne l'évolution des allongements du boulon $n^{\circ} 1$ en fonction du temps ou de l'éloignement du front.

On constate une mise en traction rapide au cours des 2 premiers jours. Ensuite la traction diminue progressivement en fonction du temps et de l'éloignement du front. Le boulon $n^{\circ} 2$ a un comportement semblable mais du fait de sa situation inclinée par rapport à la stratification, il ne fournit qu'une partie des renseignements donnés par le boulon $n^{\circ} 1$

D'autres essais « in situ » sont en cours et montrent le bon fonctionnement de ces boulons.

\section{Conclusions}

Les essais au laboratoire et in situ ont confirmé les qualités des boulons de mesures mis au point au CERCHAR : précision, fiabilité et robustesse. Ces instruments sont donc particulièrement bien adaptés à la mesure dans les conditions souvent difficiles rencontrées dans les chantiers.
Les difficultés rencontrées au cours des études antérieures ont été surmontées et nous sommes en mesure d'aborder I'influence des caractéristiques géométriques - diamètre, longueur- et mécaniques - nature de la tige - des boulons.

D'un point de vue pratique, ces boulons peuvent servir à vérifier la qualité d'un schéma de boulonnage dans un site donné et, par suite, à proposer des améliorations à ce schéma.

Notre programme d'études en cours aborde simultanément ces deux aspects.

\section{Référence Bibliographique}

[1] J. BACHACOU, B. LALLEMENT. - Contribution à l'étude du comportement des voies boulonnees en terrains houillers. Thèse Nancy, 1972. 